\title{
GDL-III: A Description Language for Epistemic General Game Playing
}

\author{
Michael Thielscher \\ School of Computer Science and Engineering \\ University of New South Wales, Australia \\ mit@unsw.edu.au
}

\begin{abstract}
GDL-III, a description language for general game playing with imperfect information and introspection, supports the specification of epistemic games. These are characterised by rules that depend on the knowledge of players. GDL-III provides a simpler language for representing actions and knowledge than existing formalisms: domain descriptions require neither explicit axioms about the epistemic effects of actions, nor explicit specifications of accessibility relations. We develop a formal semantics for GDL-III and demonstrate that this language, despite its syntactic simplicity, is expressive enough to model the famous Muddy Children domain. We also show that it significantly enhances the expressiveness of its predecessor GDL-II by formally proving that termination of games becomes undecidable, and we present experimental results with a reasoner for GDL-III applied to general epistemic puzzles.
\end{abstract}

\section{Introduction}

General game-playing (GGP) systems can understand the rules of new strategy games at runtime and learn to play these games effectively without human intervention [Genesereth $e t$ al., 2005]. The interest for AI lies in the fact that general gameplaying expertise requires algorithms beyond those designed in advance for specific games [Genesereth and Thielscher, 2014], exemplified through the annual international GGP competition since 2005 [Genesereth and Björnsson, 2013].

The language GDL, which uses the syntax and semantics of logic programming, has become the standard for describing the rules of games to general game-playing systems [Genesereth et al., 2005]. The extension GDL-II has been developed with the aim to include general imperfectinformation games [Schiffel and Thielscher, 2014]. Several general game-playing systems have recently been built to play this broader class of games too [Edelkamp et al., 2012; Schofield and Thielscher, 2015]. However, even the extended language does not support the specification of games with epistemic goals [Ågotnes et al., 2013] or, more generally, with rules that depend on the epistemic state of players. For even though we can mathematically reason about knowledge of players in imperfect-information games, it is not possible to refer to knowledge within a GDL-II rule. An example requiring this extended expressiveness are the socalled Russian Cards Problems [van Ditmarsch et al., 2006; Cordón-Franco et al., 2013], in which two cooperating players want to inform each other about their hands without a third player being able to learn anything from their (public) communication. GDL-II lacks the means to express such games since goal rules cannot refer to the knowledge of players.

With the addition of a single new keyword, the recently proposed GDL-III (for: GDL with imperfect information and introspection [Thielscher, 2016]) provides a description language suitable for epistemic general game playing. The additional keyword can be used to express individual, possibly nested knowledge, e.g. that player A knows that her cards are known to player B, as well as common knowledge. More generally, the extension to epistemic game rules is necessary in all applications of general game playing for domains where the goal of multiple agents is to gain, share and hide knowledge [Cooper et al., 2016]. GDL-III provides a simpler language for representing actions and knowledge than existing formalisms like, for example, DEL (Dynamic Epistemic Logic [Bolander and Andersen, 2011]), in that neither explicit axioms about the epistemic effects of actions are required, nor explicit specifications of accessibility relations.

In this paper, we will formally define the semantics of GDL-III. It highlights the added expressiveness that players' knowledge can influence the state transition system for the game, a feature that is not available in GDL-II. We will formally prove that termination of games becomes undecidable in GDL-III, which shows that this language goes significantly beyond the expressiveness of its predecessor.

While the main motivation for the extended language is to describe of epistemic games for the purpose of general game playing, we will also present experimental results where GDL-III is used to encode, and automatically solve, epistemic puzzles such as "Cheryl's Birthday" [Chang, 2015]. Notably, this does not require any game-playing intelligence beyond the ability to compute legal playouts based on the game rules.

\section{General Game Playing With GDL}

The Game Description Language (GDL) is a formal language for specifying the rules of strategy games to a general gameplaying system [Genesereth et al., 2005]. It uses a prefixvariant of the syntax of logic programs along with the follow- 


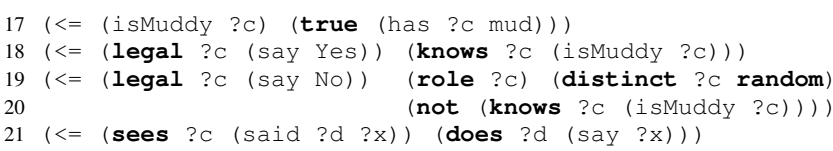

Figure 2: The remaining GDL-III game rules for MUDDYCHILDREN (without termination or goal rules).

Example 1 (cont'd) The new keyword is used in Figure 2 to complete the description of MUDDYCHILDREN: Every child must say Yes if, and only if, they know that they themselves are muddy (lines 17-20); and the children hear each other's announcement (rule 21).

The example nicely illustrate a unique feature of GDL-III as a knowledge representation language for epistemic domains: Only objective rules need to be given, which specify what agents observe and can do. While these rules implicitly determine what agents can know in principle, as we will show in this this section, it is left entirely to general game-playing systems themselves to reason about what the game rules imply about their and the other players' knowledge.

The new keyword uses reification, whereby a defined predicate, $\mathrm{P}$, is used as an argument of another predicate. ${ }^{1} \mathrm{~A}$ simple syntactic requirement ensures that nesting of knowledge is not circular: There must be some ordering $>$ on all predicate symbols $P$ that occur as argument of knows such that $P>Q$ whenever $P$ itself depends on (knows $R Q$ ) or (knows Q) [Thielscher, 2016].

The extended language GDL-III can be used to describe common epistemic imperfect-information games to general game-playing systems. An example are Russian Card Problems, where a winning condition for player Alice is that it is common knowledge that player Cath does not know anyone else's cards [Cordón-Franco et al., 2013]. In GDL-III,

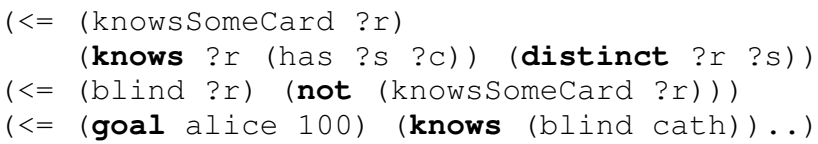

The interested reader can find the complete GDL-III description of a Russian Cards game in an accompanying technical report [Thielscher, 2017], along with a Public Announcement game [Ågotnes and van Ditmarsch, 2011] as another example.

We now define a formal semantics for GDL-III in two stages. First, we extend the logical interpretation of a set of game rules according to Definition 1 by incorporating the encoding of a given set $K=\left\{\left(\right.\right.$ knows $\left.r_{1} p_{1}\right), \ldots,\left(\right.$ knows $\left.r_{n} p_{m}\right)$, (knows $\left.q_{1}\right), \ldots,\left(\right.$ knows $\left.\left.q_{n}\right)\right\}$ of instances of the knowledge predicate. ${ }^{2}$ Most components of a game in GDL-III are evaluated relative to a given set $K$.

\footnotetext{
${ }^{1}$ Nested knowledge can be expressed with the help of auxiliary predicates; for example, (knows a knows_b_p) along with $(<=$ knows_b_p (knows $b \quad \mathrm{p})$ ) says that a knows that $\mathrm{b}$ knows $\mathrm{p}$. Syntactic sugar could of course be used to allow for actual nesting of knows handled by preprocessing.

${ }^{2}$ Here and in the following, we assume that knowledge sets $K$ are always restricted to the relevant instances of knows-expressions, that is, which occur in some rule in the underlying GDL-description.
}

Definition 2 The pre-semantics of a GDL-III game description $G$ is given by sets $R, s_{0}$ as in Definition 1 along with

- $t=\left\{(S, K): G \cup S^{\text {true }} \cup K \mid=\right.$ terminal $\}$

- $l=\left\{(r, m, S, K): G \cup S^{\text {true }} \cup K \models \operatorname{legal}(r, m)\right\}$

- $u(M, S, K)=\left\{f: G \cup M^{\text {does }} \cup S^{\text {true }} \cup K \models \operatorname{next}(f)\right\}$

- $\mathcal{I}=\left\{(r, M, S, K, p): G \cup M^{\text {does }} \cup S^{\text {true }} \cup K \models \operatorname{sees}(r, p)\right\}$

- $g=\left\{(r, v, S, K): G \cup S^{\text {true }} \cup K \models \operatorname{goal}(r, v)\right\}$

Example 1 (cont'd) Suppose the current game state is given as $S=\{$ (has bob mud) $\}$.

If $K=\{\}$, then (bob, ( say No) $, S, K) \in l$ according to rule 19-20. This would also be Bob's only legal move when $K=\{($ knows ann (isMuddy bob)), (knows ken (isMuddy bob)) $\}$, by rules 18-20.

But if, say, $K^{\prime}=\{($ knows bob (isMuddy bob)) $\}$, then game rule 18 implies that (bob, (say Yes), $\left.S, K^{\prime}\right) \in l$.

It is important to note that the pre-semantics merely tells us how to determine all game-specific predicates relative to some knowledge state. Determining the correct $K$ is the purpose of the second step in the definition of the semantics for GDL-III.

This second step requires an inductive characterisation of legal play sequences and their resulting knowledge states. Common knowledge is defined using the notion of the reflexive, transitive closure $\sim^{+}$of a given family of indistinguishability relations $\sim_{r}$ (one for every role $r$ in $R$ ). Formally, $\sim^{+}$is the smallest relation such that for all $\delta, \delta^{\prime} \delta^{\prime \prime}$ :

- $\delta \sim+\delta$ and

- if $\delta \sim^{+} \delta^{\prime}$ and $\delta^{\prime} \sim_{r} \delta^{\prime \prime}$ for some $r \in R$ then $\delta \sim^{+} \delta^{\prime \prime}$.

Definition 3 Let $G$ be a game description along with all the sets and relations it describes according to Definition 2.

- The play sequence of length 0 , denoted by $\varepsilon$, is legal and satisfies $\varepsilon \sim_{r} \varepsilon$, for all $r \in R$. It results in state $s_{0}$ and knowledge state $K_{\varepsilon}$ as the smallest set that satisfies

$$
\begin{aligned}
K_{\varepsilon}= & \left\{(\text { knows } r p): r \in R, G \cup s_{0}^{\text {true }} \cup K_{\varepsilon} \models p\right\} \\
& \cup\left\{(\text { knows } p): G \cup s_{0}^{\text {true }} \cup K_{\varepsilon}=p\right\}^{3}
\end{aligned}
$$

- For the inductive definition, let $\delta$ be a legal play sequence of length $n \geq 0$ resulting in $\left(s_{n}, K_{n}\right)$.

Sequence $\delta$ followed by $M$, written $\delta M$, is a legal play sequence of length $n+1$ if $\left(M(r), s_{n}, K_{n}\right) \in l$ for all $r \in R$. It results in state $s_{\delta M}=u\left(M, s_{n}, K_{n}\right)$ and, as the knowledge state, the smallest $K_{\delta M}$ that satisfies

$$
\begin{aligned}
K_{\delta M}=\{(\text { knows } r p): & G \cup s_{\delta^{\prime} M^{\prime}}^{\mathrm{true}} \cup K_{\delta M}=p \\
& \text { for all } \left.\delta^{\prime} M^{\prime} \sim_{r} \delta M\right\} \\
\cup\{(\text { knows } p): & G \cup s_{\delta^{\prime} M^{\prime}}^{\mathrm{true}} \cup K_{\delta M}=p \\
& \text { for all } \left.\delta^{\prime} M^{\prime} \sim^{+} \delta M\right\}
\end{aligned}
$$

This definition uses a straightforward generalisation from GDL-II of the notion of (in-)distinguishable sequences (of length $n+1$ ): Relation $\delta M \sim_{r} \delta^{\prime} M^{\prime}$ holds if role $r$ cannot distinguish $\delta$ from $\delta^{\prime}$ and takes the same move and obtains the same percepts in M. Formally,

\footnotetext{
${ }^{3}$ Hence, as in GDL-II there is no uncertainty about the initial state. However, any desired initial epistemic model can be obtained by just one random move [Ruan and Thielscher, 2014].
} 


$$
\begin{aligned}
& \text { - } \delta \sim_{r} \delta^{\prime} \\
& \text { - } M(r)=M^{\prime}(r) \\
& \text { - }\left\{p:\left(r, M, s_{n}, K_{n}, p\right) \in \mathcal{I}\right\} \\
& \quad=\left\{p^{\prime}:\left(r, M^{\prime}, s_{n}^{\prime}, K_{n}^{\prime}, p^{\prime}\right) \in \mathcal{I}\right\}
\end{aligned}
$$

This is well-defined for any GDL-III game description $G$ with acyclically defined knowledge predicates: $K_{\delta, M}$ can be "constructed" by, first, evaluating all (knows $r p$ ) and (knows $p$ ) instances for which $p$ itself does not depend on knows and, then, evaluating the other instances in accordance with the hierarchy. This implies that knowledge states are always consistent. It is also easy to verify that Definitions 2 and 3 always produce a single (knowledge) state transition system.

Definition 3 can be understood as follows: Players know everything that follows from the initial state $\left(K_{\varepsilon}\right)$. Legality and effects of moves are determined inductively from actual state $s_{n}$ and knowledge state $K_{n}$. As in GDL-II, two legal play sequences $\delta M$ and $\delta^{\prime} M^{\prime}$ cannot be distinguished by role $r$ after the last move if they were indistinguishable beforehand (i.e., $\delta \sim_{r} \delta^{\prime}$ ) and if the player made the same legal move in both $M$ and $M^{\prime}$ and obtained the same percepts. This (in-)distinguishability relation in turn determines the evaluation of the knowledge predicates, including common knowledge, for the resulting knowledge state $K_{\delta M}$.

Example 1 (cont'd) For the sake of brevity, we only show the (non-elementary) 2-children variant of our example game (with ann and bob). Let the three possible joint actions in the initial round (cf. 5-8 in Figure 1) be denoted by 01, 11, 10. Since the game rules are common knowledge, all children know that at least one of them is muddy after the first round. Nothing is observed as a direct consequence of this choice by random, however. Hence the knowledge state, which initially is empty (no one is muddy and ignoring other knowledge), does not change in any of the cases:

$$
K_{\epsilon}=\{\}=K_{01}=K_{11}=K_{10}
$$

According to the rules in Figure 2, the two children can only say No, denoted by NN below. However, by game rule 16 they get to see each other's forehead, which determines the following (in-)distinguishability after the next round:

$$
01 \cdot \mathrm{NN} \sim_{\mathrm{ann}} 11 \cdot \mathrm{NN} \sim_{\mathrm{bob}} 10 \cdot \mathrm{NN}
$$

In particular, Bob can distinguish $01 \cdot \mathrm{NN}$ from $11 \cdot \mathrm{NN}$ after receiving an information token about Ann's being muddy; similarly for Ann. From (1) in Definition 3 and relation (2), along with game rules $12-15$ and 17, it follows that

$$
\begin{aligned}
& K_{01 \cdot \mathrm{NN}}=\{(\text { knows ann (isMuddy bob) }) \text {, } \\
& \text { (knows bob (isMuddy bob)) }\} \\
& K_{11 \cdot \mathrm{NN}}=\{(\text { knows ann (isMuddy bob) }), \\
& \text { (knows bob (isMuddy ann)) }\} \\
& K_{10 \cdot \mathrm{NN}}=\{(\text { knows ann (isMuddy ann })) \text {, } \\
& \text { (knows bob (isMuddy ann)) \} }
\end{aligned}
$$

Hence, without having seen his own forehead, Bob can (in fact, must) say Yes in $01 \cdot \mathrm{NN}$; the same for Ann in $10 \cdot \mathrm{NN}$; and both can only say No in 11 . NN. Now, according to rule 21, Ann can hear what Bob says. She can thus distinguish $01 \cdot \mathrm{NN} \cdot \mathrm{NY}$ from $11 \cdot \mathrm{NN} \cdot \mathrm{NN}$, where Y abbreviates ( say Yes). Likewise,
Bob can distinguish $10 \cdot \mathrm{NN} \cdot \mathrm{YN}$ from $11 \cdot \mathrm{NN} \cdot \mathrm{NN}$. All play sequences thus become distinguishable by both players, hence:

$$
\begin{aligned}
& K_{11 \cdot \mathrm{NN} \cdot \mathrm{NN}}=\{(\text { knows ann (isMuddy } a n n)), \\
& \text { (knows ann (isMuddy bob)), } \\
& \text { (knows bob (isMuddy ann)), } \\
& \text { (knows bob (isMuddy bob)) \} }
\end{aligned}
$$

To summarise, if only one of the children is muddied by the initial random move, then he or she will know after the next round. If both are muddy, they will know one round later.

The argument can be easily generalised to prove that if $l \geq 1$ of $k$ children are muddied, then it takes $l+1$ rounds for all children to know who is muddy, for any $l \leq k$ and $k \in \mathbb{N}$.

\section{On the Expressiveness of GDL-III}

The two-stage, inductive semantics for GDL-III is considerably different from the state-transition systems for its two predecessor languages, which can be defined without induction. This indicates that the addition of the epistemic keyword significantly enhances the expressiveness of the game description language. Indeed, as we will show in the following, extending GDL-II to GDL-III has the somewhat surprising effect that termination of games becomes undecidable even under the usual syntactic restrictions that guarantee finiteness of the state space.

The intuitive reason for this is that even over a finite game state space, the knowledge that players have of each other, and of each other's knowledge, can grow arbitrarily. This is of course true for GDL-II games as well. But game rules in that language can only be conditioned on the actual game state and not the knowledge of the players, which suffices to guarantee decidability of termination. In contrast, in GDL-III the end of a game may be conditioned on the epistemic structure. In order to formally prove that this leads to the undecidability of termination, we adapt a technique introduced by Bolander and Andersen [2011] of representing a Turing machine and its tape with the help of an epistemic structure.

Let an arbitrary Turing machine (TM) be given, with finite set of states $Q$, binary alphabet $\{$ blank, marked and initially blank tape. We construct a GDL-III game with random and two players, called ann and bob, as follows. The game states are built from the finite set of features $Q \cup\{$ blank, marked, (right ann), (right bob) $\}$. The initial game state is encoded by rule 2 in Figure 3, where $\mathrm{q} 0 \in Q$ is the starting state of the TM.

Each instruction of the Turing machine is translated to a small set of legal moves for random. Moves by random are only partially observed by the two players, thus leading to a new knowledge structure that corresponds to the next state of the Turing machine and its tape. As an example, Figure 3 shows the encoding for transitions of the form

$$
(q, \text { blank }) \rightarrow\left(q^{\prime}, \text { marked, right }\right)
$$

Put in words, if the TM is in state $q$ and over a blank cell, then the cell gets marked, the TM transitions to state $q^{\prime}$ and the head moves to the right. This instruction gets translated into a set of eight possible moves, abbreviated as ( $i$ ? 1 ? x) in Figure 3 with $? r \in\{a n n, b o b\}$ and $? x \in\{1 \ldots 4\}$. 

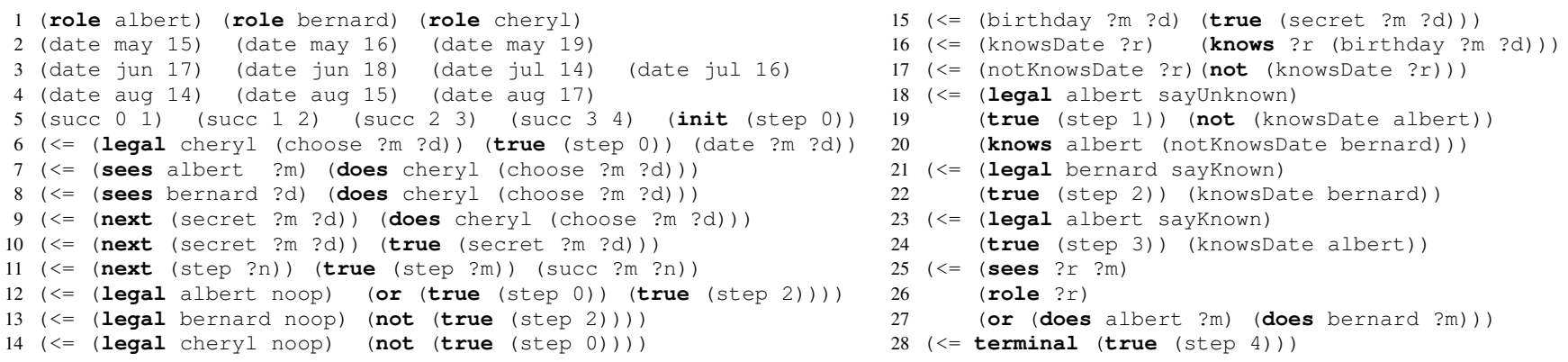

Figure 4: CherylsBirThDAY: a possible description of this puzzle using the syntax of GDL with introspection. Cheryl begins by picking a date (rule 6), of which Albert and Bernard only get to see the month and day, respectively (lines 7-8). Using three defined properties (lines 15-17), announcements are modelled by two moves (sayUnknown, sayKnown) whose preconditions assume players to be truthful (lines 18-24) and which are public (rule 25-27).

with http://potassco. sourceforge. net, an offthe-shelf answer set solver, for interpreting GDL-III rules. Times are reported in seconds (CPU time). The original problem consists of 10 dates across 4 different months and 6 different days. We kept a similar ratio of different "months" and "days" as we increased the problem size (\# of dates) in order to ensure that the randomly chosen instances are equally difficult in that the number of solutions averages to approximately one. ${ }^{7}$ The results are summarised in the table below for each size and averaged over 1,000 random problem instances. They demonstrate how the average time to compute legal play sequences increases since this game requires maintaining and evaluating the knowledge state of both roles, including what they entail about one player's knowledge of the other player.

\begin{tabular}{|l|l|l||l|l|}
\hline \#dates & \#months & \#days & avg \#solutions & avg time \\
\hline \hline 10 & 5 & 5 & 0.57 & 0.00 \\
\hline 100 & 30 & 30 & 1.37 & 0.01 \\
\hline 1000 & 200 & 200 & 1.07 & 0.20 \\
\hline 10000 & 1500 & 1500 & 0.68 & 3.79 \\
\hline
\end{tabular}

\section{Related Work and Conclusion}

The extended general game description language GDL-III shares with its predecessor GDL-II the unique feature that game designers have to specify only the objective rules about what players can see and do. Rules about how moves and percepts affect the knowledge of players are not required. Our semantics of the knowledge operator in GDL-III inherits the concept of indistinguishability of play sequences used to characterise the evolution of knowledge in GDL-II, assuming players with perfect recall [Schiffel and Thielscher, 2014]. Knowledge preconditions and knowledge goals in GDL-III therefore refer to what players can and cannot know in principle given the observations they make throughout a game.

The added expressiveness necessitates a semantics where state transition systems, which provide the full semantics for GDL-II, act merely as a pre-semantics. The complete semantics for GDL-III accounts for knowledge feeding back into the

\footnotetext{
${ }^{7}$ Cheryl's Birthday would of course be less famous if it did not have a unique solution, but problems with no or multiple solutions are equally relevant for testing the runtime behaviour of a controller.
}

definition of legal play sequences. This significantly enhances the expressiveness of game descriptions, but it also leads to the undecidability of termination of games in general. This does not affect the practicality of the language for general game playing, however. Since all reachable (epistemic) states are finite, all reasoning problems, such as determining legal moves, remain decidable. A game designer can easily guarantee termination of a game, e.g. through the standard use of a step counter [Genesereth and Björnsson, 2013].

Our experiments have shown how the size of the information sets of players influences the runtimes of a basic reasoner, unlike in case of GDL-II. Explicitly maintaining the set of relevant legal play sequences is practically viable for short epistemic games or puzzles. However, many games will require compact representations of information sets, e.g. as has been proposed for Kriegspiel [Ciancarini and Favini, 2007], or approximations via state sampling [Long et al., 2010].

Several languages for epistemic multi-agent domains have been proposed recently, including [Kominis and Geffner, 2015; Muise et al., 2015; Jiang et al., 2016]. These differ from GDL-III in that they all require explicit specifications of what an agent knows (respectively, believes) as the result of an action or sensing. To axiomatise MUDDYCHILDREN in any of these languages, for instance, you would need to provide explicit axioms on the effect of a child (not) saying "yes" on everyone else's knowledge. Likewise, epistemic puzzles similar to Cheryl's Birthday have been solved using model checking systems for Dynamic Epistemic Logic [van Ditmarsch et al., 2005]. Again, the main difference is that DEL requires an explicit encoding of an epistemic structure in form of a concrete accessibility relation for the problem in question.

The proposed encoding of epistemic puzzles uses GDL different from its main purpose in general game playing, because no players actually play the game. Yet it is an interesting by-product of having a basic reasoner for the new language element. GDL-III also has applications in general game playing beyond the description of epistemic games. For example, it could be used by GDL-II players to generate logical strategy rules by which they condition their moves on their knowledge. Beyond general game playing, GDL-III can be used for general multi-agent epistemic problems [Cooper et al., 2016]. 


\section{References}

[Ågotnes and van Ditmarsch, 2011] Thomas Ågotnes and Hans van Ditmarsch. What will they say?-Public announcement games. Synthese, 179:57-85, 2011.

[Ågotnes et al., 2013] Thomas Ågotnes, Paul Harrenstein, Wiebe van der Hoek, and Michael Wooldridge. Boolean games with epistemic goals. In Logic, Rationality, and Interaction, volume 8196 of LNCS, pages 1-14, Hangzhou, China, October 2013. Springer.

[Bolander and Andersen, 2011] Thomas Bolander and Mikkel Andersen. Epistemic planning for single- and multi-agent systems. Journal of Applied Non-Classical Logics, 21(1):9-34, 2011.

[Chang, 2015] Kenneth Chang. A math problem from Singapore goes viral: When is Cheryl's birthday? The New York Times, 14 Apr 2015.

[Ciancarini and Favini, 2007] Paolo Ciancarini and Gian Piero Favini. Representing kriegspiel states with metapositions. In Proceedings of the International Joint Conference on Artificial Intelligence (IJCAI), pages 2450-2455, Hyderabad, India, January 2007.

[Cooper et al., 2016] Martin Cooper, Andreas Herzig, Faustine Maffre, Frédeéric Maris, and Pierre Régnier. A simple account of multi-agent epistemic planning. In Proceedings of the European Conference on Artificial Intelligence (ECAI), pages 193-201, The Hague, August 2016.

[Cordón-Franco et al., 2013] Andrés Cordón-Franco, Hans van Ditmarsch, David Fernández Duque, and Fernando Soler-Toscano. A colouring protocol for the generalized Russian cards problem. Journal of Theoretical Computer Science, 495:81-95, 2013.

[Edelkamp et al., 2012] Stefan Edelkamp, Tim Federholzner, and Peter Kissmann. Searching with partial belief states in general games with incomplete information. In Proceedings of the German Annual Conference on Artificial Intelligence (KI), volume 7526 of $L N C S$, pages 25-36, Saarbrücken, Germany, September 2012. Springer.

[Genesereth and Björnsson, 2013] Michael Genesereth and Yngvi Björnsson. The international general game playing competition. AI Magazine, 34(2):107-111, 2013.

[Genesereth and Thielscher, 2014] Michael Genesereth and Michael Thielscher. General Game Playing. Synthesis Lectures on Artificial Intelligence and Machine Learning. Morgan \& Claypool, 2014.

[Genesereth et al., 2005] Michael Genesereth, Nathaniel Love, and Barney Pell. General game playing: Overview of the AAAI competition. AI Magazine, 26(2):62-72, 2005.

[Jiang et al., 2016] Guifei Jiang, Dongmo Zhang, Laurent Perrussel, and Heng Zhang. Epistemic GDL: A logic for representing and reasoning about imperfect information games. In Proceedings of the International Joint Conference on Artificial Intelligence (IJCAI), pages 1138-1144, New York, 2016.
[Kominis and Geffner, 2015] Filippos Kominis and Hector Geffner. Beliefs in multiagent planning: From one agent to many. In Proceedings of the International Conference on Automated Planning and Scheduling (ICAPS), pages 147-155, Jerusalem, Israel, June 2015.

[Long et al., 2010] Jeffrey Long, Nathan Sturtevant, Michael Buro, and Timothy Furtak. Understanding the success of perfect information Monte Carlo sampling in game tree search. In Proceedings of the AAAI Conference on Artificial Intelligence, pages 134-140, Atlanta, July 2010.

[Love et al., 2006] Nathaniel Love, Timothy Hinrichs, David Haley, Eric Schkufza, and Michael Genesereth. General Game Playing: Game Description Language Specification. Technical Report LG-2006-01, Stanford University, 2006. Available at: games. stanford.edu.

[Muise et al., 2015] Christian Muise, Vaishak Belle, Paolo Felli, Sheila McIlraith, Tim Miller, Adrian Pearce, and Liz Sonenberg. Planning over multi-agent epistemic states: A classical planning approach. In Proceedings of the AAAI Conference on Artificial Intelligence, pages 3327-3334, Austin, January 2015.

[Ruan and Thielscher, 2014] Ji Ruan and Michael Thielscher. Logical-epistemic foundations of general game descriptions. Studia Logica, 102(2):321-338, 2014.

[Schiffel and Thielscher, 2014] Stephan Schiffel and Michael Thielscher. Representing and reasoning about the rules of general games with imperfect information. Journal of Artificial Intelligence Research, 49:171-206, 2014.

[Schofield and Thielscher, 2015] Michael Schofield and Michael Thielscher. Lifting model sampling for general game playing to incomplete-information models. In Proceedings of the AAAI Conference on Artificial Intelligence, pages 3585-3591, Austin, January 2015.

[Thielscher, 2016] Michael Thielscher. GDL-III: A proposal to extend the game description language to general epistemic games. In Proceedings of the European Conference on Artificial Intelligence (ECAI), pages 1630-1631, The Hague, August 2016.

[Thielscher, 2017] Michael Thielscher. A formal description language for general epistemic games. Technical Report UNSW-CSE-TR-201708, School of Computer Science and Engineering, University of New South Wales, May 2017. Available at: www.cse.unsw. edu . au/ reports /

[van Benthem, 2014] Johan van Benthem. Logic in Games. MIT Press, 2014.

[van Ditmarsch et al., 2005] Hans van Ditmarsch, Ji Ruan, and Rineke Verbrugge. Model checking Sum and Product. In Proceedings of the Australasian Joint Conference on Artificial Intelligence, volume 3809 of LNCS, pages 790-795, Sydney, Australia, December 2005. Springer.

[van Ditmarsch et al., 2006] Hans van Ditmarsch, Wiebe van der Hoek, Ron van der Meyden, and Ji Ruan. Model checking russian cards. Electronic Notes in Theoretical Computer Science, 149(2):105-123, 2006. 